\title{
The analysis of combustion heat of the metallized solid fuel compositions
}

\author{
Yana Dubkova, ${ }^{1, *}$, and Alexander Zhukov ${ }^{1}$ \\ ${ }^{1}$ National Research Tomsk State University, 634050 Tomsk, Russia
}

\begin{abstract}
The technique and results of the pilot study of power characteristics of the solid-fuel compositions containing metal additives are shown. Results of the comparative analysis of influence of various additives on the caloric content of fuel compositions are given.
\end{abstract}

\section{Introduction}

Fuel for the modern aircraft has to answer a big number of requirements: to have high specific impulse, high density, the required aggregate state of components under operating conditions, has to be stable, safe in the address, nontoxical, compatible to constructional materials, have raw material resources etc. These conditions, in turn, provides reliable and long operation of aircraft. Along with above-mentioned one of the major power characteristics is combustion heat or caloric content of fuel Q. Combustion heat (caloric content) of material is defined as amount of thermal energy which is emitted at the complete combustion of a mass unit of fuel [1], [Q] $=\mathrm{J} / \mathrm{kg}$ or $[\mathrm{Q}]=\mathrm{a} \mathrm{cal} / \mathrm{kg}$. Now much attention is paid to development of high-energy materials (HEM). For effective use of HEM it is necessary to know their main characteristics. The technique and results of a research of combustion heat of the exemplars made of new compositions of high-energy materials are given in this work.

\section{Materials and methods of the experimental research}

Exemplars of the mixed solid fuels [2] containing in quality combustible-binding divinyl rubber of the SKDM- 80 brand as an oxidizer - bidispersny powder of perchlorate of PHA ammonium / were experimentally investigated by. For increase in power characteristics fuel compositions powders of diboride of a titanium $\mathrm{TiB}_{2}, \mathrm{AlB}_{2}$ dialuminium boride, amorphous pine forest $\mathrm{B}, \mathrm{AlB}_{12}$ polyaluminium boride, powder of aluminum of the ASD-4 brand were entered. Component structures of the studied HEM are brought in table 1

Table 1. Component structures of the studied HEMs.

\begin{tabular}{|c|c|c|c|c|c|}
\hline \multirow{2}{*}{ Component of HEM, mass \% } & \multicolumn{5}{|c|}{ No. HEM sample } \\
\cline { 2 - 6 } & 1 & 2 & 3 & 4 & 5 \\
\hline SKDM-80 & 14.8 & 13.6 & 11.0 & 11.8 & 16.0 \\
\hline
\end{tabular}

\footnotetext{
* Corresponding author: y.a.dubkova@,niipmm.tsu.ru
} 


\begin{tabular}{|c|c|c|c|c|c|}
\hline Ammonium perchlorate & 70.2 & 71.4 & 74.0 & 73.2 & 69.0 \\
\hline $\mathrm{TiB}_{2}$ & 15.0 & - & - & - & - \\
\hline $\mathrm{AlB}_{2}$ & - & 15.0 & - & - & - \\
\hline $\mathrm{B}$ & - & - & 15.0 & - & - \\
\hline $\mathrm{AlB}_{12}$ & - & - & - & 15.0 & - \\
\hline $\mathrm{ASD}-4$ & - & - & - & - & 15.0 \\
\hline
\end{tabular}

Measurement of combustion heat of the studied exemplars of high-energy materials is taken on calorimetric installation [3]. A basic element of calorimetric installation is the bomb calorimeter. Schemes of a calorimeter and a bomb calorimeter are provided on figures 1,2 . In the figure 3, a), b) the general view of installation and a bomb calorimeter are given.

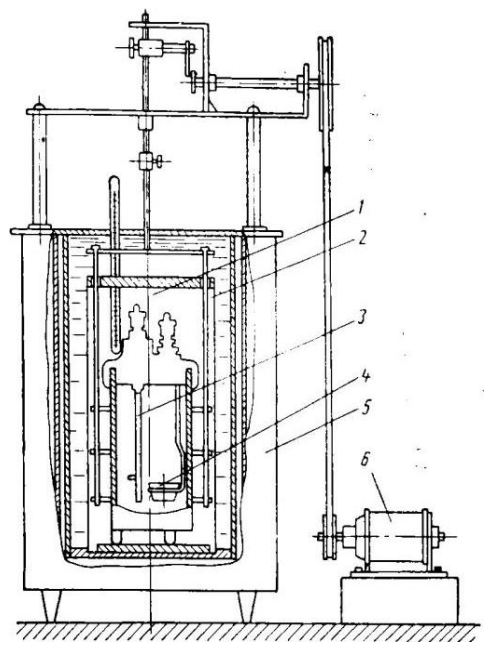

Fig. 1. The scheme of calorimetric installation for definition of combustion heat of fuel: 1 camera, 2 mixer, a 3- flame igniter, 4 crucible with the examinee fuel, 5 housing of a calorimeter, 6 electric motor.

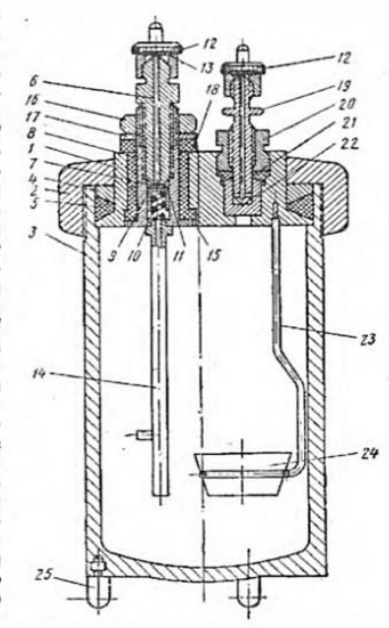

Fig. 2. Scheme of a bomb calorimeter: 1cover; 2-tightening ring; 3 - glass; 4 - metal ring; 5 - rubber ring; 6, 19 - the union; 7 ftoroplastovy laying; 8 - housing; 9 cylindrical valve; 10 - spring; 11 - aluminum laying; 12 - removable caps; 13 - ftoroplastovy laying; 14 - electrocontact tube; 15 - ebonite insulator; 16 - nut; 17 - washer; 18 - ebonite laying; 20 - omental nut; 21 - epiploon; 22 saddle; 23 - current carrying pin; 24 - cup; 25 cylinder foot. 


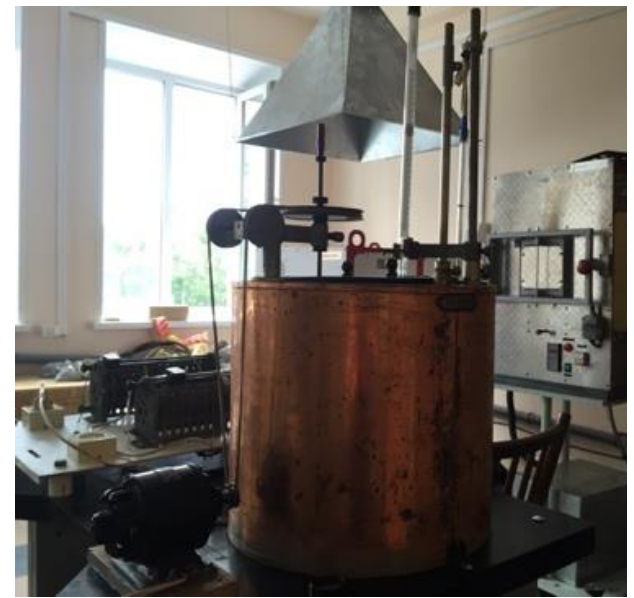

a

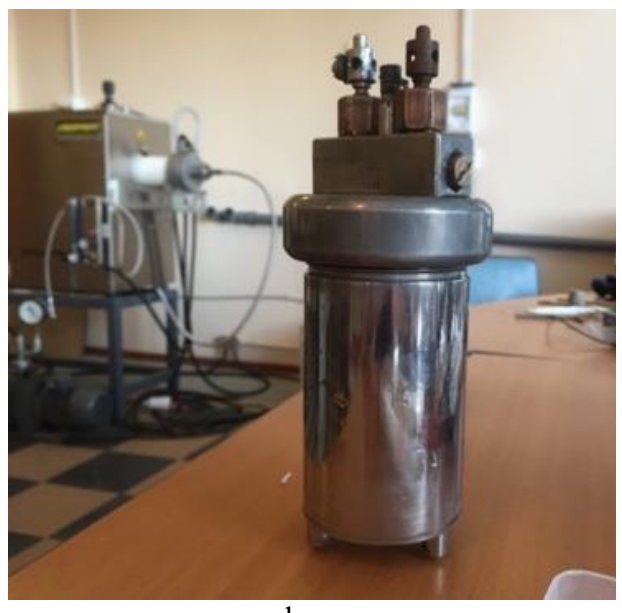

b

Fig. 3. A general view of a calorimeter (a) and a bomb calorimeter (b) for measurement of combustion heat of HEM.

Solid-fuel elements were carried out in the form of cylinders (fig. 4). In the course of manufacture of exemplars entered a hardener in number of 0.5 masses $\%$ into powder system over the mass of mix.

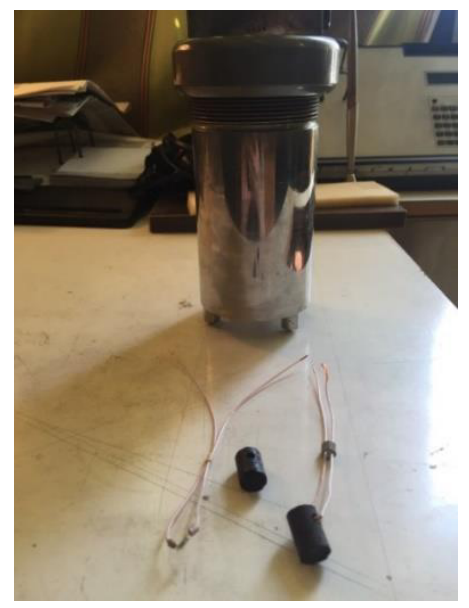

Fig. 4. HEM samples prepared for placement in a calorimeter bomb.

Before carrying out experement exemplars measured, weighed then prepared them for landing in a calorimeter bomb. In a vessel of a calorimeter poured distilled water, lowered a bomb and took water temperature the metastatic Beckmann thermometer (fig. 5 and), b), c) allowing to receive a high precision of measurements. After the executed measurements ignition and step-by-step measurement of water temperature in a calorimeter was carried out. Calibration was carried out by combustion of standard solid fuel of ballistidny typ ("N" blasting powder) with the known combustion heat [4]. 


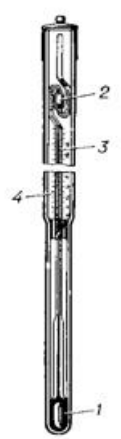

a)

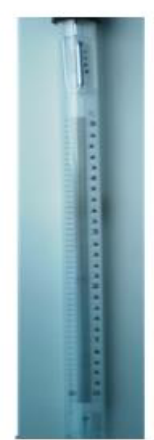

b)

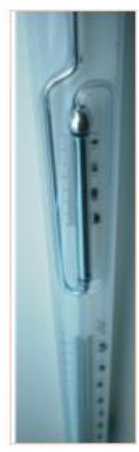

c)

Fig. 5. Beckman thermometer (a): 1 - tank; 2 - padding camera; 3 - capillary; 4 - main scale; (b), (c) - a general view.

According to theoretical bases of the experimental technique, the total of the warmth which is marked out at combustion of the studied HEM exemplar with the mass of $\mathrm{m}$ is determined by a formula

$$
Q^{\prime}=c_{p}(M+W+B)(\Delta T \pm \Delta \theta)
$$

where $W=\frac{c_{k}}{c_{p}} M_{k}$ is a thermal capacity (water equivalent) of a calorimeter; $B=\frac{c_{\mathrm{B}}}{c_{p}} M_{k}$ is a allowance for availability of air in a calorimeter; $\Delta T=T_{k}-T_{n}$.

Combustion heat $Q$ fuel is determined by a formula

$$
Q=\frac{Q^{\prime}}{m}=\frac{c_{p}(M+W+B)(\Delta T \pm \Delta \theta)}{m}
$$

The mass of water in calorimetric installation makes $M=3000 \mathrm{~g}$; water equivalent of a calorimeter of $\mathrm{W}=449 \mathrm{~g}, \mathrm{~B}=1.219 \mathrm{~g}$. Assessment of extent of correction of $\mathrm{B}$ in a formula (2) on availability of air in a calorimeter when determining caloric content of fuel on a formula (3) showed that at change of temperature and pressure in actual measuring ranges $\mathrm{T}=(283 \div 303) \mathrm{K}, \mathrm{p}=(740 \div 780)$ millimetre of mercury the mass of air in a calorimeter changes slightly. The maximal difference of extent of correction of B from its value under normal conditions makes $\sim 5 \%$. At the same time the mass of air in a calorimeter makes $0.06 \%$ of cooperative mass of water and a water equivalent of the device. Therefore, in the considered conditions the air contribution (extent of correction of B) when determining caloric content of fuel on a formula (2) is negligible, and the formula (2) can be presented in the following form:

$$
Q=\frac{Q^{\prime}}{m}=\frac{c_{p}(M+W)(\Delta T \pm \Delta \theta)}{m}
$$

The sign "+" or "-" in (3) corresponds to heating or cooling of the device owing to heat exchange with a surrounding medium.

Taking into account the aforesaid the formula (2) can be presented in the form

$$
Q^{\prime}=c_{p}(M+W) \Delta T \pm c_{p}(M+W) \Delta \theta
$$

where a summand in a right member is the amendment to definition of value of combustion heat on heat exchange of calorimetric installation with a surrounding medium. 
The accounting of heat exchange of calorimetric installation with a surrounding medium when determining combustion heat of fuel on a formula (2) is carried out on the basis of the comparative analysis of reference value of combustion heat $Q_{r e f}^{\prime}$ and results of measurement of the quantity of heat $Q^{\prime}{ }_{\text {instr }}$ allocated at combustion of reference HEM. The total of warmth which is allocated at combustion of a standard sample of HEM with the mass of $\mathrm{m}$ of $\mathrm{g}$ is determined by a formula

$$
Q_{r e f}^{\prime}=Q_{r e f} \cdot m
$$

where $Q_{\text {ref }}$ is the caloric content of reference HEM the known value.

Mean value of allowance for heat exchange of the device with a surrounding medium is determined by results of a series of the experiments put on reference HEM ("N" blasting powder). Results of measurement of combustion heat of reference HEM are given in table 2 .

Table 2. Results of measurement of combustion heat of reference HEM ("N" blasting powder).

\begin{tabular}{|c|c|c|c|c|c|c|}
\hline No & $m, \mathrm{~g}$ & $\Delta T, \mathrm{~K}$ & $Q_{r e f}^{\prime}$, cal & $Q_{\text {instr }}^{\prime}$, cal & $\Delta Q^{\prime}, \mathrm{cal}$ & $Q_{\text {real }}, \mathrm{cal} / \mathrm{g}$ \\
\hline 1 & 2.520 & 0.76 & 2142 & 2621 & 479 & 862 \\
\hline 2 & 2.400 & 0.72 & 2040 & 2483 & 443 & 847 \\
\hline 3 & 2.416 & 0.73 & 2054 & 2518 & 463 & 856 \\
\hline 4 & 2.349 & 0.70 & 1997 & 2414 & 417 & 836 \\
\hline \multicolumn{5}{|c|}{ Average } & 450 & 850 \\
\hline
\end{tabular}

Thus, on the basis of results of measurement of combustion heat $Q_{\text {real }}$ of reference HEM value of allowance for heat exchange of calorimetric installation with a surrounding medium $\Delta \overline{Q^{\prime}}=-450$ cal feces is received. Substituting in (3) received value and values of sizes corresponding to parameters of the unit, the working formula for calculation of combustion heat of the studied HEM has an appearance:

$$
Q=\frac{c_{p}(M+W) \cdot \Delta T-\Delta \bar{Q}^{\prime}}{m}=\frac{3449 \cdot \Delta T-450}{m},
$$

where $[\Delta T]=\mathrm{K} ;[M]=\mathrm{g} ;[Q]=\mathrm{cal} / \mathrm{g}$.

\section{Results}

The results of measurement of combustion heat of the view HEM received on the basis of the offered technique are given in table 3.

\begin{tabular}{|c|c|c|c|c|c|}
\hline No. sample & $m, \mathrm{~g}$ & $\Delta T, \mathrm{~K}$ & $Q_{i n s t r}^{\prime}$, cal & $Q, \mathrm{cal} / \mathrm{g}$ & $\bar{Q}, \mathrm{cal} / \mathrm{g}$ \\
\hline \multirow{2}{*}{1.} & 2.250 & 0.87 & 3001 & 1134 & \multirow{2}{*}{1126} \\
\hline & 2.065 & 0.80 & 2759 & 1118 & \\
\hline \multirow{2}{*}{2.} & 1.720 & 0.81 & 2794 & 1363 & \multirow{2}{*}{1338} \\
\hline & 1.917 & 0.86 & 2966 & 1312 & \\
\hline \multirow{2}{*}{3.} & 1.410 & 0.83 & 2863 & 1711 & \multirow{2}{*}{1476} \\
\hline & 2.250 & 0.94 & 3242 & 1240 & \\
\hline \multirow{2}{*}{4.} & 1.880 & 0.98 & 3380 & 1559 & \multirow{2}{*}{1534} \\
\hline & 2.079 & 1.04 & 3587 & 1509 & \\
\hline
\end{tabular}

Table 3. Combustion heat of exemplars of HEM. 


\begin{tabular}{|l|l|l|l|l|l|}
\hline \multirow{2}{*}{5.} & 1.797 & 0.84 & 2897 & 1362 & \multirow{2}{*}{1352} \\
\cline { 2 - 5 } & 2.004 & 0.91 & 3139 & 1342 & \\
\hline
\end{tabular}

In table 4 the received values of combustion heat of exemplars of HEM are given in the International System of Units.

Table 4. Combustion heat of samples of HEM.

\begin{tabular}{|c|c|c|c|c|c|c|}
\hline \multirow{3}{*}{$\mathrm{Q}, \mathrm{MJ} / \mathrm{kg}$} & \multicolumn{7}{|c|}{ No. sample } \\
\cline { 2 - 7 } & 1 & 2 & 3 & 4 & 5 & Reference \\
\cline { 2 - 7 } & 4.72 & 5.61 & 6.18 & 6.43 & 5.66 & 3.56 \\
\hline
\end{tabular}

\section{Conclusion}

The method and results of the pilot study of combustion heat of the solid-fuel compositions containing various metal additives are presented $\left(\mathrm{TiB}_{2}, \mathrm{AlB}_{2}, \mathrm{AlB}_{12}, \mathrm{Al}, \mathrm{B}\right)$. The tmethod is based on use of a calorimetric way of definition of combustion heat of materials. By results of a series of the experiments put on reference HEM ("N" blasting powder), an allowance for defined value heat exchange $\Delta \overline{Q^{\prime}}$ of a calorimeter with a surrounding medium.

On the basis of the offered technique combustion heat of high-energy materials with additives of metal powders is measured. The comparative analysis of influence of effectiveness of various additives on fuel caloric content is carried out. It is received that the maximal heat effect is reached at combustion of $\mathrm{HEM}$ containing $\mathrm{AlB}_{12}$ polyaluminium boride (exemplar No. 4).

The work was financially supported by the Ministry of Education and Science of the Russian Federation within the framework of the Federal Target Program. Agreement No. 14.578.21.0034 (Unique identifier RFMEFI57814X0034).

\section{References}

1. Ya. M. Shapiro, G. Yu. Mazing, N. E. Prudnikov, The theory of the rocket engine on solid fuel (Voyenizdat, Moscow, 1966) [in Russian]

2. V. A Arkhipov., A. V Pesterev., L. A. Savelyeva, R. A. Yusupov, Higher Education Institutions. Physics. 9, 8 (2012)

3. K. N. Godovskaya, L. V. Ryabina, G.Yu. Novik, M. M. Gerner, Commercial analysis (The higher school, Moscow, 1972) [in Russian]

4. A. P. Babichev, N. A. Babushkin, A. M. Bratkovsky, Physical quantities: Reference book, (Energoatom, Moscow, 1991) [in Russian] 